${ }^{1}$ Departamento de Enfermedades Respiratorias. Facultad de Medicina, Pontificia Universidad Católica de Chile. Santiago, Chile. ${ }^{2}$ Departamento de Radiología. Hospital Clínico Universidad de Chile. Santiago, Chile. ${ }^{3}$ Servicio de Medicina. Hospital de Carabineros. Santiago, Chile. anterna de Medicina, Facultad de Medicina, Pontificia Universidad Católica de Chile.

Fuente de financiamiento: Proyecto Fondecyt 1080671.

Recibido el 9 de julio de 2015 , aceptado el 3 de noviembre de

Correspondencia a: Dr. Fernando Saldías Peñafiel Departamento de Enfermedades Respiratorias, División de Medicina, Facultad de Medicina, Pontificia Universidad Católica de Chile.

Marcoleta 350, Santiago, Chile. Teléfonos: (562) 26331541

(562) 23543242 Fax: (562) 26335255 fsaldias@med.puc.cl

\section{Detección precoz de cáncer pulmonar con tomografía computarizada de tórax en pacientes con enfermedad pulmonar obstructiva crónica tabáquica}

\author{
FERNANDO SALDÍAS P. ${ }^{1}$, JUAN CARLOS DÍAZ P. ${ }^{2}$, CARMEN RAIN M.a \\ PAMELA ILLANES C. ${ }^{a}$, RODRIGO DÍAZ T. ${ }^{3}$, ORLANDO DÍAZ P. ${ }^{1}$

\section{Early detection of lung cancer using computed tomography among patients with chronic obstructive pulmonary disease}

Background: Chest computed tomography (CT) scan may improve lung cancer detection at early stages in high risk populations. Aim: To assess the diagnostic performance of chest CT in early lung cancer detection in patients with chronic obstructive pulmonary disease (COPD). Patients and Methods: One hundred sixty one patients aged 50 to 80 years, active or former smokers of 15 or more pack-years and with COPD were enrolled. They underwent annual respiratory functional assessment and chest computed tomography for three years and were followed for five years. Results: Chest CT allowed the detection of lung cancer in nine patients (diagnostic yield: 5.6\%). Three cases were detected in the initial CT and six cases in follow-up scans. Most patients were in early stages of the disease ( 6 stage Ia and 1 stage $I b$ ). Two patients were diagnosed at advanced stages of the disease and died due to complications of cancer. Two thirds of patients had nonspecific pulmonary nodules on the initial chest CT scan (100 patients, 62\%). Seventy four percent had less than three nodules and were of less than $5 \mathrm{~mm}$ of diameter in 57\%. In 92\% of cases, these were false positive findings. In the follow-up chest CT, lung nodules were detected in two thirds of patients and $94 \%$ of cases corresponded to false positive findings. Conclusions: Chest CT scans may detect lung cancer at earlier stages in COPD patients.

(Rev Med Chile 2016; 144: 202-210)

Key words: Early diagnosis; Lung neoplasms; Obstructive; Prognosis; Pulmonary disease, chronic; Tomography, X-Ray.
$\mathrm{E}$ 1 cáncer pulmonar es la neoplasia que afecta más individuos en el mundo desde hace varias décadas, con una incidencia estimada de 1,8 millones de casos el año 2012, correspondiendo a $12,9 \%$ del total ${ }^{1}$. La mayoría de los casos son pesquisados en etapas avanzadas de la enfermedad (etapas III y IV), limitando nuestras alternativas terapéuticas (cirugía, quimioterapia y radioterapia), determinando su elevada letali- dad (15\% de sobrevida a 5 años) $)^{2}$. Es la principal causa de muerte por neoplasia en el mundo, se estima que fallecieron 1,59 millones de pacientes debido a esta dolencia en 2012, correspondiendo a $19,4 \%$ de las muertes por cáncer ${ }^{1}$. En Estados Unidos de Norteamérica, es la tercera neoplasia más frecuente en hombres y mujeres, siendo la principal causa de muerte, determinando 27\% de las muertes relacionadas a neoplasia ${ }^{2,3}$. En 
Chile, en el año 2011 fallecieron 2.780 personas por esta neoplasia, correspondiendo a una tasa de mortalidad de 16,1 por 100.000 habitantes y 2,9\% de las causas de mortalidad general ${ }^{4}$. El 85\% de los casos están relacionados con el consumo de tabaco, siendo mayor el riesgo en fumadores pesados (más de $30 \mathrm{paq} / \mathrm{año}$ ), ex-fumadores recientes (menos de 15 años de abstención) y en portadores de enfermedad pulmonar obstructiva crónica (EPOC) $)^{5-11}$. Según la Encuesta Nacional de Salud, 53,4\% (IC 95\% 51,1-55,7\%) de la población chilena es o ha sido fumadora alguna vez en su vida, predominando en varones y en los estratos educacionales medio-alto ${ }^{12}$.

Los programas de detección precoz de cáncer pulmonar en población de riesgo, empleando la radiografía de tórax y estudio citológico de la expectoración, no han demostrado que reduzcan la mortalidad asociada a esta neoplasia ${ }^{13}$. Sin embargo, la tomografía computarizada (TC) de tórax con multidetector ha permitido obtener imágenes volumétricas de alta resolución con niveles aceptables de radiación, lo que permitiría detectar el cáncer pulmonar en estadios más precoces de la enfermedad ${ }^{14}$. Estudios observacionales han demostrado que la TC de tórax de baja dosis permitiría detectar más pacientes con cáncer pulmonar en estadios precoces (etapas I y II), susceptibles de tratamiento quirúrgico, comparado con la radiografía de tórax ${ }^{15}$. El ensayo clínico aleatorizado financiado por el Instituto Nacional del Cáncer de los Estados Unidos de Norteamérica (National Lung Screening Trial) enroló a 53.454 sujetos entre 55 y 74 años, fumadores o ex-fumadores de al menos 30 paq/año, para ingresar a un programa de pesquisa de cáncer pulmonar mediante TC de tórax o radiografía de tórax anual durante tres años $^{16}$. Este estudio demostró una reducción de 20\% (IC 95\% 6,8 a 26,7\%; p: 0,004) en el riesgo de morir por cáncer de pulmón en el grupo que fue evaluado con tomografía de tórax comparado con la radiografía de tórax. Sin embargo, se triplicó $(24,2 \%$ vs $6,9 \%)$ la pesquisa de nódulos pulmonares de aspecto benigno que requirieron seguimiento con imágenes y ocasionalmente estudios invasivos (biopsia pulmonar percutánea, transbronquial o quirúrgica).

El propósito de este estudio es examinar el rendimiento diagnóstico de la tomografía computarizada de tórax en la pesquisa de cáncer de pulmón en una cohorte de pacientes portadores de enfermedad pulmonar obstructiva crónica tabáquica.

\section{Pacientes y Métodos}

Estudio de cohorte prospectivo de seguimiento a seis años (enero de 2008 a diciembre de 2014) que examinó las variables clínicas, imagenológicas y funcionales asociadas a la limitación de la actividad física en pacientes con EPOC tabáquica $^{17}$. Se evaluaron 161 pacientes fumadores o ex-fumadores portadores de EPOC tabáquica de magnitud leve a muy severa, definida de acuerdo a los criterios de GOLD ${ }^{18}$, en condiciones estables de su enfermedad, quienes aceptaron participar en el protocolo firmando un acta de consentimiento informado aprobada por el Comité de Ética de nuestra institución. Los criterios de inclusión fueron: adultos entre 50 y 80 años con EPOC ( $\mathrm{VEF}_{1} / \mathrm{CVF}$ post broncodilatador menor a $70 \%$ ), con historia de tabaquismo de 15 o más paquetes año, ser fumador activo o haber dejado de fumar en los últimos quince años y no tener alguna enfermedad que les impidiera realizar la evaluación funcional respiratoria o que por su gravedad no pudieran completar el período de seguimiento. Se excluyó a los pacientes con historia de asma bronquial, portadores de bronquiectasias, secuelas de tuberculosis, enfermedad neoplásica conocida, inmunodeficiencia, comorbilidades descompensadas, resección pulmonar, tratamiento con esteroides sistémicos en los últimos tres meses, historia de hemoptisis, dolor torácico o baja de peso mayor de $8 \mathrm{~kg}$ en los últimos doce meses de causa desconocida.

En todos los pacientes incorporados al estudio, durante la fase estable de la enfermedad, se consignó las características sociodemográficas, consumo de tabaco (expresado en paquetes/año), las comorbilidades, peso, talla e IMC, se evaluó la magnitud de la disnea utilizando la escala modificada del Medical Research Council del Reino Unido $^{19}$ (mMRC) y se registró el número de exacerbaciones en los últimos 12 meses, de acuerdo a la definición de Burge y Wedzicha ${ }^{20}$.

Se realizó una TC helicoidal de tórax en todos los pacientes al ser enrolados en el estudio y luego en el seguimiento a los 12 y 24 meses. Dos radiólogos involucrados en el estudio examinaron las imágenes en forma independiente e informaron 
los hallazgos significativos: pesquisa de nódulos y masas (número, tamaño, localización, textura [sólido, en vidrio esmerilado], características de los bordes, presencia de calcificaciones), adenopatías hiliares y mediastínicas mayores de $1 \mathrm{~cm}$, presencia de opacidades intersticiales o alveolares, derrame pleural, engrosamiento pleural, u otros hallazgos (cardiomegalia, calcificaciones de arterias coronarias o pared aórtica, enfermedad de vía aérea pequeña, bronquiectasias, hernia hiatal). El examen tomográfico se realizó con el paciente en inspiración profunda, con un equipo multidetector de 64 canales (Somatom Sensation 64, Siemens Healthcare, Erlangen, Alemania), que fue calibrado diariamente para la densidad tomográfica del aire y cada tres meses para el agua. El protocolo de adquisición de imágenes fue el siguiente: 120 kVp, 200 mAs y tiempo de rotación de 0,33 segundos. Las imágenes fueron reconstruidas usando el algoritmo B70, con cortes de $1 \mathrm{~mm}$ de grosor y $0,7 \mathrm{~mm}$ de intervalo. La magnitud del enfisema se estimó midiendo el porcentaje de áreas de baja atenuación inferior a -960 Unidades Hounsfield $(\% \text { LAA-960) })^{17}$.

El examen tomográfico inicial o de seguimiento fue considerado positivo si se pesquisaron nódulos no calcificados de más de $3 \mathrm{~mm}$ de diámetro, masas en el parénquima pulmonar, o hubo crecimiento de los nódulos en el seguimiento a los 12 ó 24 meses. En los nódulos inespecíficos inferiores a $5 \mathrm{~mm}$ de diámetro se sugirió seguimiento tomográfico durante dos años, en los nódulos mayores de $10 \mathrm{~mm}$, o si se objetivó crecimiento del nódulo en el seguimiento, se recomendó estudio invasivo (biopsia percutánea bajo TC, transbronquial o quirúrgica) ${ }^{21}$. En los nódulos de tamaño intermedio se recomendó realizar seguimiento tomográfico o estudio invasivo de acuerdo a los factores de riesgo del paciente y su reserva cardiopulmonar. En la evaluación del riesgo de neoplasia de los pacientes se consideró las características tomográficas de los nódulos, la condición de fumador activo o ex-fumador, la magnitud del consumo de tabaco expresado en paquetes año, la duración de la suspensión del hábito tabáquico expresada en años y los antecedentes personales y familiares de neoplasia.

En el seguimiento a 60 meses, se consignaron las neoplasias broncogénicas pesquisadas mediante tomografía, su variedad histológica, estadio clínico y tratamiento.
Se realizó una espirometría con un equipo Sensor Medics 2200 antes y después de la administración de salbutamol, siguiendo las normas de las Sociedades Americana de Tórax (ATS) y Europea de Enfermedades Respiratorias (ERS) ${ }^{22}$. Los valores se expresaron utilizando los valores de referencia de Hankinson y cols ${ }^{23}$. Se efectuó una prueba de caminata en 6 min de acuerdo a las normas de la $\mathrm{ATS}^{24}$. Para esta última prueba se emplearon los valores de referencia de Troosters y cols ${ }^{25}$. Durante la caminata se registró la $\mathrm{SpO}_{2} \mathrm{y}$ frecuencia cardiaca con oxímetro de pulso (Nonin Medical Inc., Plymouth, Mn., EE. UU.). La disnea y fatigabilidad de las piernas se evaluaron empleando la escala psicofísica de Borg ${ }^{26}$. Se calculó el índice de gravedad multidimensional $\mathrm{BODE}^{27}$, que incluye el IMC, $\mathrm{VEF}_{1}$, magnitud de la disnea y distancia recorrida en $6 \mathrm{~min}$.

El seguimiento clínico de la cohorte de pacientes finalizó el 31 de diciembre de 2014, las principales fuentes de información fueron: a) Ficha clínica ambulatoria; b) Registro de hospitalizaciones; c) Contacto vía telefónica; d) Informes del Registro Civil. Los datos de sobrevida de la cohorte fueron obtenidos del registro civil y no hubo pérdidas en el seguimiento a 60 meses.

Los resultados son expresados como valores promedio \pm desviación estándar y rangos para las variables medidas en escala numérica y en porcentaje para las medidas en escala nominal.

\section{Resultados}

Se enrolaron 161 pacientes con EPOC tabáquica en la cohorte de seguimiento con tomografía computarizada de tórax durante dos años consecutivos (Tabla 1). La edad promedio de los pacientes era $65,7 \pm 8,8$ años (rango: $50-80$ ), $60 \%$ varones, dos tercios ex-fumadores y un tercio fumadores activos, consumo de tabaco promedio de $48 \pm 24$ paq/año (rango: 15-150), 84\% tenía comorbilidades, especialmente cardiovasculares y metabólicas crónicas, cerca de la mitad presentaban tos y expectoración crónica y limitación significativa en la actividad física debido a la disnea $(m M R C \geq 2)$. Un tercio de los pacientes estaban en las etapas III y IV de GOLD, 6,2\% eran usuarios de oxígeno domiciliario, $20 \%$ tenía sobrepeso, $57 \%$ había presentado una o más exacerbaciones de la EPOC en los últimos 12 meses, siendo 
Tabla 1. Características clínicas de la cohorte de pacientes con enfermedad pulmonar obstructiva crónica

\begin{tabular}{|c|c|c|}
\hline Características & $n(\bar{x} \pm D E)$ & $\%$ (Rango) \\
\hline $\mathrm{n}$ & 161 & 100 \\
\hline Edad (años) & $65,7 \pm 8,8$ & (R: $50-80)$ \\
\hline Género M-F & $96-65$ & $60-40$ \\
\hline Exfumador-fumador activo & $105-56$ & $65-35$ \\
\hline Consumo de tabaco (paq/año) & $48,6 \pm 24,3$ & (R: 15-150) \\
\hline Tos y expectoración crónica & 88 & 54,7 \\
\hline Disnea $(m M R C \geq 2)$ & 90 & 55,9 \\
\hline Área de enfisema (-960 UH) & $15,8 \pm 10,6$ & (R: 0,8-57,9) \\
\hline Exac. EPOC $\leq 12$ meses & $1,4 \pm 1,7$ & (R: 0-10) \\
\hline $\mathrm{IMC}\left(\mathrm{Kg} / \mathrm{m}^{2}\right)$ & $26,5 \pm 4,2$ & (R: 16-38) \\
\hline Distancia recorrida 6 min (m) & $474 \pm 103$ & (R: 80-712) \\
\hline Índice BODE & $2,2 \pm 2,1$ & (R: 0-10) \\
\hline Comorbilidades & $135 / 161$ & 83,9 \\
\hline Cardiopatía coronaria & 12 & 7,5 \\
\hline Hipertensión arterial & 83 & 51,6 \\
\hline Diabetes mellitus & 27 & 16,8 \\
\hline Dislipidemia & 58 & 36,0 \\
\hline Reflujo gastroesofágico & 57 & 35,4 \\
\hline Obesidad & 33 & 20,5 \\
\hline Depresión & 19 & 11,8 \\
\hline CVF (mL) & $3.380 \pm 1.010$ & $(99 \pm 20 \%$ teórico $)$ \\
\hline $\operatorname{VEF}_{1}(\mathrm{~mL})$ & $1.640 \pm 790$ & (62 $\pm 25 \%$ teórico) \\
\hline $\mathrm{VEF}_{1} / \mathrm{CVF}(\%)$ & $47,9 \pm 15,3$ & (R: 16-68) \\
\hline GOLD I-II-III-IV & $50-51-38-22$ & $31-32-23-14$ \\
\hline Sobrevida a 60 meses & $127 / 161$ & $78,9 \%$ \\
\hline
\end{tabular}

Nota: M: Masculino, F. Femenino, Exac. EPOC $\leq 12$ meses: Exacerbaciones de la EPOC en últimos 12 meses, IMC: Índice de masa corporal, CVF: Capacidad vital forzada, $V_{1} F_{1}$ : Volumen espiratorio forzado del primer segundo.

23\% exacerbaciones graves. La mayoría de los pacientes estaban siendo tratados con agonistas $\beta$ adrenérgicos o anticolinérgicos de acción larga $(68 \%)$, corticoides inhalados (59\%) y agonistas $\beta_{2}$ adrenérgicos o anticolinérgicos de acción corta (72\%). Treinta y cuatro pacientes fallecieron en el seguimiento a 60 meses (letalidad: $21 \%$ ).

Dos tercios de los pacientes presentaban nódulos pulmonares inespecíficos en la tomografía computarizada inicial (100 pacientes, 62\%), la mayoría tenía menos de tres nódulos (74\%) y eran menores de $5 \mathrm{~mm}$ de diámetro (57\%). En la tomografía inicial, 92\% de los pacientes con nódulos pulmonares correspondieron a lesiones no neoplásicas en el seguimiento a cinco años, detectándose alteraciones significativas en ocho pacientes con cáncer pulmonar. En las tomografías de seguimiento se pesquisaron nódulos pulmonares en dos tercios de los pacientes, siendo 94\% de los casos falsos positivos. En esta cohorte de seguimiento no hubo procedimientos invasivos o toracotomías en pacientes con nódulos benignos. 
La tomografía computarizada de tórax permitió detectar a nueve pacientes con cáncer pulmonar (rendimiento diagnóstico: 5,6\%) en el seguimiento a 24 meses, la mayoría en etapas precoces de la enfermedad (6 etapa Ia y 1 etapa Ib); sin embargo, sólo dos pacientes fueron sometidos a cirugía con fines curativos, sin evidencias de recidiva de la neoplasia en el seguimiento a cinco años (Tabla 2). Cuatro pacientes tenían contraindicaciones para la cirugía debido a la pobre reserva cardiopulmonar, tres eran usuarios de oxígeno domiciliario, quienes se mantuvieron estables en el seguimiento clínico, objetivándose lento crecimiento de la lesión tumoral. Una paciente fue tratada con radioterapia ablativa estererotáxica logrando control local de la lesión y se mantiene estable en el seguimiento a cinco años; otra paciente fue pesquisada en etapas avanzadas de la enfermedad (etapa IIIb), siendo tratada con quimioterapia (etopósido + cisplatino) y radioterapia, falleciendo por complicaciones re- lacionadas a la neoplasia (metástasis pulmonares y pleurales) a los 35 meses de seguimiento. Un paciente fue pesquisado en estadíos avanzados de la enfermedad, con metástasis mediastínicas y pulmonares contralaterales, falleciendo a los 7 meses de seguimiento. La tomografía inicial permitió pesquisar a tres pacientes con neoplasia pulmonar (prevalencia: 1,9\%) y las tomografías de seguimiento demostraron la aparición o crecimiento de nódulos pulmonares malignos en seis pacientes (incidencia: 3,7\%).

Los hallazgos incidentales en la tomografía torácica se describen en la Tabla 3, destacando las lesiones neoplásicas extrapulmonares (tres casos), enfermedades intersticiales asociadas o no al consumo de tabaco ( 8 casos), enfermedades de la vía aérea (bronquiolitis: 7 y bronquiectasias: 13) y un grupo misceláneo (calcificaciones de arterias coronarias y aorta, bulas, granulomas calcificados, lesiones cicatriciales, atelectasias, hernia hiatal).

Tabla 2. Pesquisa de cáncer pulmonar mediante tomografía computarizada de tórax de dosis convencional en pacientes portadores de enfermedad pulmonar obstructiva crónica

\begin{tabular}{|c|c|c|c|c|}
\hline Edad, Sexo & $\begin{array}{l}\text { Etapa GOLD } \\
\text { Tabaquismo }\end{array}$ & $\begin{array}{l}\text { Características del } \\
\text { nódulo }\end{array}$ & $\begin{array}{l}\text { Histología } \\
\text { Estadío clínico }\end{array}$ & $\begin{array}{l}\text { Tratamiento } \\
\text { Sobrevida a } 5 \text { años }\end{array}$ \\
\hline 72 años, F & III - 62 paq/año & Língula, 1,6 cm espiculado & $\begin{array}{l}\text { No precisada } \\
\text { Etapa la }\end{array}$ & $\begin{array}{l}\text { PRCP*-Seguimiento } \\
\text { Vivo a } 60 \text { meses }\end{array}$ \\
\hline 71 años, F & I - 45 paq/año & LSD, 1,8 cm espiculado & $\begin{array}{l}\text { Adenocarcinoma } \\
\text { Etapa la }\end{array}$ & $\begin{array}{l}\text { Lobectomía LSD } \\
\text { Vivo a } 60 \text { meses }\end{array}$ \\
\hline 78 años, M & II - 150 paq/año & LID, 1,5 cm espiculado & $\begin{array}{l}\text { No precisada } \\
\text { Etapa la }\end{array}$ & $\begin{array}{l}\text { PRCP*-Seguimiento } \\
\text { Vivo a } 60 \text { meses }\end{array}$ \\
\hline 73 años, M & II - 62 paq/año & LID, $2 \mathrm{~cm}$ espiculado & $\begin{array}{l}\text { Adenocarcinoma } \\
\text { Etapa la }\end{array}$ & $\begin{array}{l}\text { Lobectomía LID } \\
\text { Vivo a } 60 \text { meses }\end{array}$ \\
\hline 78 años, M & II - 53 paq/año & LSI, 1 cm espiculado & $\begin{array}{l}\text { No precisada } \\
\text { Etapa la }\end{array}$ & $\begin{array}{l}\text { PRCP-Seguimiento } \\
\text { Vivo a } 60 \text { meses }\end{array}$ \\
\hline 62 años, F & I - 80 paq/año & LSD, 5,6 cm espiculado & $\begin{array}{l}\text { Ca pavimentoso } \\
\text { Etapa IIIb }\end{array}$ & $\begin{array}{l}\text { QT-RT } \\
\text { Fallece a los } 35 \text { meses }\end{array}$ \\
\hline 67 años, F & IV - 60 paq/año & LSD, 3,3 cm espiculado & $\begin{array}{l}\text { No precisada } \\
\text { Etapa Ib }\end{array}$ & $\begin{array}{l}\text { Radioterapia ablativa } \\
\text { Vivo a } 60 \text { meses }\end{array}$ \\
\hline 71 años, M & II - 60 paq/año & Língula, 2,9 cm espiculado & $\begin{array}{l}\text { No precisada } \\
\text { Etapa la }\end{array}$ & $\begin{array}{l}\text { PRCP*-Seguimiento } \\
\text { Vivo a } 60 \text { meses }\end{array}$ \\
\hline 70 años, M & I - 38 paq/año & LSI, 6,4 cm espiculado & $\begin{array}{l}\text { Ca pavimentoso } \\
\text { Etapa IV }\end{array}$ & $\begin{array}{l}\text { Tratamiento paliativo } \\
\text { Fallece a los } 7 \text { meses }\end{array}$ \\
\hline
\end{tabular}

Nota: M: Masculino, F: Femenino, LSD: Lóbulo superior derecho, LID: Lóbulo inferior derecho, LSI: Lóbulo superior izquierdo. Ca: Carcinoma. PRCP: Pobre reserva cardiopulmonar basada en estudios funcionales (espirometría, DLCO, gases arteriales, cintigrafía pulmonar y test cardiopulmonar), $\left(^{*}\right)$ : Usuario de oxígeno domiciliario. 
Detección precoz de cáncer de pulmón por TC en EPOC - F. Saldías et al

Tabla 3. Hallazgos incidentales en la tomografía computarizada de tórax realizada a pacientes con enfermedad pulmonar obstructiva crónica

\begin{tabular}{|c|c|c|c|}
\hline Hallazgos & Características & $\mathbf{n}$ & Evolución \\
\hline \multirow[t]{3}{*}{ Neoplasias } & Mesotelioma pleural & 1 & $\begin{array}{l}70 \text { años, Tumor irresecable } \\
\text { Fallece a los } 14 \text { meses }\end{array}$ \\
\hline & Tumor fibroso de la pleura & 1 & $\begin{array}{l}60 \text { años, Tumor resecado } \\
\text { Vivo a } 60 \text { meses }\end{array}$ \\
\hline & Hepatocarcinoma & 1 & $\begin{array}{l}70 \text { años, Tumor resecado } \\
\text { Vivo a } 60 \text { meses }\end{array}$ \\
\hline \multirow[t]{2}{*}{ Enfermedades de la vía aérea } & Bronquiectasias & 13 & \\
\hline & Bronquiolitis & 7 & \\
\hline \multirow[t]{2}{*}{ Enf. pulmonar difusa } & Fibrosis pulmonar asociada a EPOC & 4 & \\
\hline & Enfermedad intersticial asociada a tabaquismo & 4 & \\
\hline Vasculares & Calcificaciones arterias coronarias y aorta & 25 & \\
\hline \multirow[t]{6}{*}{ Misceláneas } & Lesiones cicatriciales & 11 & \\
\hline & Granulomas calcificados & 10 & \\
\hline & Atelectasias & 14 & \\
\hline & Bulas & 9 & \\
\hline & Engrosamiento pleural & 6 & \\
\hline & Hernia hiatal & 4 & \\
\hline
\end{tabular}

Tabla 4. Estudios clínicos controlados de detección precoz de cáncer pulmonar mediante tomografía computarizada de tórax de baja dosis en población de riesgo elevado. Análisis: Mortalidad por cáncer pulmonar ${ }^{16,28-30}$

\begin{tabular}{|c|c|c|c|c|c|c|c|}
\hline $\begin{array}{l}\text { Estudios, } \\
\text { año }\end{array}$ & $\mathbf{n}$ & $\begin{array}{c}\text { Edad } \\
\text { (años) } \\
\text { M (\%) }\end{array}$ & $\begin{array}{l}\text { Consumo } \\
\text { de tabaco } \\
\text { (paq/año) }\end{array}$ & $\begin{array}{l}\text { Intervalos de } \\
\text { screening y } \\
\text { mediana de } \\
\text { seguimiento } \\
\text { (años) }\end{array}$ & $\begin{array}{c}\text { Pesquisa } \\
\text { de nódulos } \\
\text { pulmonares } \\
(\%)\end{array}$ & $\begin{array}{c}\text { Pesquisa } \\
\text { de nódulos } \\
\text { pulmonares } \\
\text { neoplásicos } \\
(\%)\end{array}$ & $\begin{array}{c}\text { Muertes } \\
\text { por 100.000 } \\
\text { personas-año } \\
\text { RR (IC } 95 \% \text { ) }\end{array}$ \\
\hline $\begin{array}{l}\text { DANTE, } \\
2009\end{array}$ & $\begin{array}{l}\text { TCT: } 1.276 \\
\text { CT: } 1.196\end{array}$ & $\begin{array}{l}60-74 \\
100 \%\end{array}$ & $\begin{array}{l}\text { TCT: } 47,3 \\
\text { CT: } 47,2\end{array}$ & $\begin{array}{c}0-1-2-3-4 \\
2,8 \text { años }\end{array}$ & $18 \%$ & $3,7 \%$ & $\begin{array}{c}\text { TCT: } 527 \\
\text { CT: } 637 \\
0,83(0,45-1,54)\end{array}$ \\
\hline $\begin{array}{l}\text { NLST, } \\
2011\end{array}$ & $\begin{array}{l}\text { TCT: } 26.722 \\
\text { Rx: } 26.732\end{array}$ & $\begin{array}{c}55-74 \\
59 \%\end{array}$ & $\begin{array}{l}\text { Promedio: } \\
56\end{array}$ & $\begin{array}{l}0-1-2 \\
6,5 \text { años }\end{array}$ & $27-28-17 \%$ & $1-0,6-0,9 \%$ & $\begin{array}{c}\text { TCT: } 247 \\
\text { Rx: } 309 \\
0,80(0,73-0,93)\end{array}$ \\
\hline $\begin{array}{l}\text { DLCST, } \\
2012\end{array}$ & $\begin{array}{l}\text { TCT: } 2.052 \\
\text { CT: } 2.052\end{array}$ & $\begin{array}{c}50-70 \\
55 \%\end{array}$ & $\begin{array}{l}\mathrm{TCT}: 36,4 \\
\mathrm{CT}: 35,9\end{array}$ & $\begin{array}{c}0-1-2-3-4 \\
4,8 \text { personas-año }\end{array}$ & $\begin{array}{c}9,2-5,9-2,7 \\
-4,1-3,9 \%\end{array}$ & $\begin{array}{c}0,8-0,6- \\
0,7 \%\end{array}$ & $\begin{array}{c}\text { TCT: } 154 \\
\text { CT: } 112 \\
1,37(0,63-2,97)\end{array}$ \\
\hline $\begin{array}{l}\text { MILD, } \\
2012\end{array}$ & $\begin{array}{l}\text { TCT: } 2.376 \\
\text { CT: } 1.723\end{array}$ & $\begin{array}{l}\geq 49 \\
66 \%\end{array}$ & $\begin{array}{l}\text { TCT: } 39 \\
\text { CT: } 38\end{array}$ & $\begin{array}{c}0-1-2-3-4 \\
4,4 \text { años }\end{array}$ & $15 \%$ & $2,1 \%$ & $\begin{array}{c}\text { TCT: } 216 \\
\text { CT: } 109 \\
1,99(0,80-4,96)\end{array}$ \\
\hline
\end{tabular}

Nota: DANTE: Detection and Screening of Early Lung Cancer by Novel Imaging Technology and Molecular Essays; NLST: National Lung Screening Trial; DLCST: Danish Lung Cancer Screening Trial; MILD: Multicentric Italian Lung Detection. TCT: Tomografía computarizada de tórax de baja dosis. Rx: Radiografía de tórax. CT: Grupo control. M: Sexo masculino. RR: Riesgo relativo. IC 95\%: Intervalo de confianza del 95\%. 


\section{Discusión}

Los principales hallazgos del estudio fueron: a) La tomografía computarizada de tórax fue útil en la pesquisa de cáncer pulmonar en pacientes con EPOC tabáquica; b) La mayoría de los pacientes fueron pesquisados en etapas precoces de la enfermedad (etapa I); c) La edad avanzada y pobre reserva cardiopulmonar limitaron las alternativas terapéuticas en dos tercios de los pacientes pesquisados en estadío I; d) La mayoría de los nódulos pulmonares pesquisados en la tomografía computarizada de tórax correspondieron a lesiones no neoplásicas (falsos positivos).

El rendimiento diagnóstico de la tomografía computarizada de tórax de dosis convencional en la pesquisa de cáncer pulmonar en una cohorte de pacientes con EPOC tabáquica fue 5,6\% en el seguimiento a cinco años, similar a lo descrito en los estudios de tamizaje observacionales ${ }^{13,15,21}$, siendo superior a la radiografía de tórax en los estudios controlados $^{16,28-30}$ (Tabla 4). El rendimiento del examen sería más elevado en los grandes fumadores (más de 30 paq/año), ex-fumadores recientes (menos de 15 años de abstención), portadores de enfermedad pulmonar obstructiva crónica o daño pulmonar crónico (neumoconiosis, fibrosis pulmonar) y en pacientes con antecedentes familiares de neoplasia pulmonar-11,31.

La mayoría de los pacientes fueron pesquisados en etapas precoces de la enfermedad $(77,8 \%$ de los casos en estadío I), similar a lo descrito en la literatura ${ }^{13,15,16,21,28-30}$; sin embargo, la edad avanzada y la pobre reserva cardiopulmonar de los enfermos fueron contraindicación para la cirugía en dos tercios de los casos. Por este motivo, la Sociedad Americana de Oncología Clínica ha recomendado evaluar los programas de detección precoz de cáncer pulmonar mediante tomografía en la población de alto riesgo, entre 55 y 74 años, fumadores o ex-fumadores recientes de más de 30 paq/año y con expectativas de vida que justifiquen esta intervención: mejorar la calidad de vida y sobrevida de los enfermos ${ }^{32}$.

Los estudios de tamizaje han demostrado la superioridad de la tomografía sobre la radiografía de tórax y la citología de esputo en la pesquisa precoz de cáncer pulmonar ${ }^{13,15}$; sin embargo, la mayoría de los estudios no han demostrado mejoría en la supervivencia probablemente debido a limitaciones metodológicas (bajas tasas de reclutamiento y menor potencia estadística), excepto el National Lung Screening Trial (NLST) realizado en Estados Unidos de Norteamérica que enroló a más de cincuenta mil individuos de alto riesgo asintomáticos entre 55 y 74 años, fumadores o ex-fumadores de al menos 30 paq/año y demostró una reducción de $20 \%$ en la mortalidad relacionada a la neoplasia en el seguimiento a mediano plazo (mediana: 6,5 años) y reducción de 6,7\% de la mortalidad global (IC 95\% 1,2-13,6\%, p: 0,02) ${ }^{16}$.

En nuestro estudio, se pesquisaron nódulos pulmonares inespecíficos en dos tercios de los pacientes con EPOC, siendo cerca de 95\% de los casos falsos positivos; similar a lo acontecido en el NLST ${ }^{16}$, donde $39,1 \%$ de los pacientes tuvo algún examen positivo en el seguimiento a tres años y 96,4\% fueron falsos positivos; siendo el valor predictivo positivo del examen de 3,6\%. La mayoría de los nódulo detectados en la tomografía son benignos (98\%) y menores de $1 \mathrm{~cm}$ de diámetro $^{15,16,28-30,33}$. Dado que el riesgo de neoplasia depende directamente del diámetro de la lesión, la textura y características de sus bordes, se han propuesto diferentes protocolos para su manejo; en los nódulos pequeños se recomienda realizar seguimiento tomográfico cada seis meses durante dos años, y si se detecta aumento en el tamaño de la lesión se recomienda realizar un examen invasivo para comprobar la existencia de células malignas $13,15,21$.

Similar a lo acontecido en los estudios de tamizaje ${ }^{15,16,28-30}$, en nuestra cohorte de pacientes con EPOC no observamos reducción significativa en la estadificación de los pacientes con cáncer de pulmón detectados en los estudios de imágenes de seguimiento comparado con el estudio inicial y el valor predictivo positivo del examen fue inferior a $5 \%$.

Los costos elevados del examen, los riesgos asociados a la radiación y el elevado porcentaje de nódulos pulmonares inespecíficos de etiología benigna detectados en las pruebas de tamizaje han determinado que la mayoría de los sistemas de salud no recomienden su uso extensivo y de rutina en las poblaciones de riesgo ${ }^{15,34,35}$. Sin embargo, en Estados unidos de Norteamérica, algunas instituciones de salud, como la United States Preventive Task Force, American College of Chest Physicians y American Society of Clinical Oncology, han comenzado a recomendar el estudio de tamizaje anual con TC de tórax de baja dosis en pacientes entre 
55 y 74 años, fumadores al menos de 30 paq/año o ex-fumadores con menos de 15 años de suspensión del consumo de tabaco ${ }^{15}$. Aún faltan los estudios de costo-efectividad y de seguimiento a largo plazo que permitan avalar esta recomendación.

\section{Referencias}

1. World Health Organization. International Agency for Research on Cancer. GLOBOCAN 2012: Estimated Cancer Incidence, Mortality and Prevalence Worldwide in 2012. Lung Cancer. Accessed June 20, 2015.

2. Centers for Disease Control and Prevention. National Center for Health Statistics. CDC WONDER On-line Database, compiled from Compressed Mortality File 1999-2012 Series 20 No. 2R, 2014.

3. American Cancer Society. Cancer Facts \& Figure 2013. Atlanta, GA: American Cancer Soc; 2013.

4. Ministerio de Salud. Gobierno de Chile. Departamento de Estadísticas e Información de Salud. Serie de mortalidad por causa 2000-2012. http://deis.minsal.cl/vitales/ Mortalidad_causa/Chile.htm

5. Strauss GM. Screening for lung cancer: an evidence-based synthesis. Surg Oncol Clin N Am 1999; 8: 747-74.

6. Osann KE. Lung cancer in women: the importance of smoking, family history of cancer, and medical history of respiratory disease. Cancer Res 1991; 51: 4893-7.

7. Burns DM. Primary prevention, smoking, and smoking cessation: implications for future trends in lung cancer prevention. Cancer 2000; 89 (11 Suppl): 2506-9.

8. Halpern MT, Gillespie BW, Warner KE. Patterns of absolute risk of lung cancer mortality in former smokers. J Natl Cancer Inst 1993; 85: 457-64.

9. Raji OY, Duffy SW, Agbaje OF, Baker SG, Christiani DC, Cassidy A, et al. Predictive accuracy of the Liverpool Lung Project risk model for stratifying patients for computed tomography screening for lung cancer: a case-control and cohort validation study. Ann Intern Med 2012; 157: 242-50.

10. Tammemagi MC, Katki HA, Hocking WG, Church TR, Caporaso N, Kvale PA, et al. Selection criteria for lung-cancer screening. N Engl J Med 2013; 368: 728-36.

11. Kovalchik SA, Tammemagi M, Berg CD, Caporaso NE, Riley TL, Korch M, et al. Targeting of low-dose CT screening according to the risk of lung-cancer death. N Engl J Med 2013; 369: 245-54.

12. Ministerio de Salud. Exposición a tabaco. Encuesta Nacional de Salud 2009-2010, Chile. Páginas 152-88.

13. Doria-Rose VP, Szabo E. Screening and prevention of lung cancer. In: Kern-stine, KH; Reckamp, KL, editors.
Lung cancer: a multidisciplinary approach to diagnosis and management. New York: Demos Medical Publishing; 2010, page 53-72.

14. Naidich DP, Marshall CH, Gribbin C, Arams RS, McCauley DI. Low-dose CT of the lungs: preliminary observations. Radiology 1990; 175: 729-31.

15. Bach PB, Mirkin JN, Oliver TK, Azzoli CG, Berry DA, Brawley OW, et al. Benefits and harms of CT screening for lung cancer: a systematic review. JAMA 2012; 307: 2418-29.

16. National Lung Screening Trial Research Team, Aberle DR, Adams AM, Berg CD, Black WC, Clapp JD, Fagerstrom RM, et al. Reduced lung-cancer mortality with low-dose computed tomographic screening. N Engl J Med 2011; 365: 395-409.

17. Díaz AA, Morales A, Díaz JC, Ramos C, Klaassen J, Saldías F, et al. CT and physiologic determinants of dyspnea and exercise capacity during the six-minute walk test in mild COPD. Respir Med 2013; 107: 570-9.

18. Global Initiative for Chronic Obstructive Lung Disease (GOLD). Global strategy for the diagnosis, management and prevention of chronic obstructive pulmonary disease 2015. www.goldcopd.com

19. Mahler DA, Wells CK. Evaluation of clinical methods for rating dyspnea. Chest 1988; 93: 580-6.

20. Burge S, Wedzicha JA. COPD exacerbations: definitions and classifications. Eur Respir J 2003; 41 (Suppl): 46S-53S.

21. Henschke CI, Yankelevitz DF, Libby DM, McCauley D, Pasmantier M, Altorki NK, et al. Early lung cancer action project: annual screening using single-slice helical CT. Ann N Y Acad Sci 2001; 952: 124-34.

22. Miller MR, Hankinson J, Brusasco V, Burgos F, Casaburi $\mathrm{R}$, Coates A, et al. Standardisation of spirometry. Eur Respir J 2005; 26: 319-38.

23. Hankinson JL, Odencrantz JR, Fedan KB. Spirometric reference values from a sample of the general U.S. population. Am J Respir Crit Care Med 1999; 159: 179-87.

24. American Thoracic Society Statement. Guidelines for the six-minute walk test. Am J Respir Crit Care Med 2002; 166: 111-7.

25. Troosters T, Gosselink S, Decramer M. Six minute walking distance in healthy elderly subjects. Eur Respir J 1999; 14: 270-4.

26. Borg GA. Psychophysical basis of perceived exertion. Med Sci Sports Exerc 1982; 14: 377-81.

27. Celli BR, Cote CG, Marín JM, Casanova C, Montes de Oca M, Méndez RA, et al. The body-mass index, airflow obstruction, dyspnea and exercise capacity index in chronic obstructive pulmonary disease. N Engl J Med 2004; 350: 1005-12. 
28. Infante M, Cavuto S, Lutman FR, Brambilla G, Chiesa G, Ceresoli G, et al. DANTE Study Group. A randomized study of lung cancer screening with spiral computed tomography: three-year results from the DANTE trial. Am J Respir Crit Care Med 2009; 180: 445-53.

29. Saghir Z, Dirksen A, Ashraf H, Bach KS, Brodersen J, Clementsen PF, et al. CT screening for lung cancer brings forward early disease. The randomized Danish Lung Cancer Screening Trial: status after five annual screening rounds with low-dose CT. Thorax 2012; 67: 296-301.

30. Pastorino U, Rossi M, Rosato V, Marchiano A, Sverzellati N, Morosi C, et al. Annual or biennial CT screening versus observation in heavy smokers: 5 -year results of the MILD trial. Eur J Cancer Prev 2012; 21: 308-15.

31. Nitadori J, Inoue M, Iwasaki M, Otani T, Sasazuki S, Nagai K, et al. Association between lung cancer inci- dence and family history of lung cancer: data from a large-scale population-based cohort study, the JPHC study. Chest 2006; 130: 968-75.

32. Jemal A, Siegel R, Xu J, Ward E. Cancer statistics, CA Cancer J Clin 2010; 60: 277-300.

33. Gierada DS, Pinsky P, Nath H, Chiles C, Duan F, Aberle DR. Projected outcomes using different nodule sizes to define a positive CT lung cancer screening examination. J Natl Cancer Inst 2014; 106 (11): dju284.

34. Humphrey LL, Deffebach M, Pappas M, Baumann C, Artis K, Mitchell JP, et al. Screening for lung cancer with low-dose computed tomography: A systematic review to update the U.S. preventive services task force recommendation. Ann Intern Med 2013; 159: 411-20.

35. Manser R, Lethaby A, Irving LB, Stone C, Byrnes G, Abramson MJ, Campbell D. Screening for lung cancer. Cochrane Database Syst Rev 201; 6: CD001991. 